Bull. Egypt. Soc. Physiol. Sci. Vol. (41) Issue (2), 155-167

\author{
Bull. of Egyp. Soc. Physiol. Sci. \\ (Official Journal of Egyptian Society for Physiological Sciences) \\ (pISSN: 1110-0842; eISSN: 2356-9514)
}

\title{
Enhancing ECG Diagnosis Using Hybrid Automated Technique
}

\author{
Mai A. Shams El-Din ${ }^{1}$, Mohamed Rizk M. Rizk ${ }^{2}$, Sherif. M. Abd Elsamad ${ }^{3}$ and Nancy Diaa $^{1}$ \\ 1Department of Biomedical Engineering, Medical Research Institute, Alexandria University, Egypt \\ 2 Department of Electrical Engineering, Faculty of Engineering, Alexandria University, Egypt \\ 3 Cardiology and Angiology Unit, Medical Research Institute, Alexandria University, Egypt
}

Submit Date: May 15, 2020

Revise Date: July 14, 2020

Available online: Jan 10, 2021

\section{Keywords}

- Wavelet

- ECG automated

Diagnosis

- Scaling technique

- ECG Computer interpretations

- Hybrid system

\section{Abstract}

The electrocardiogram (ECG) is a test of electrical activities of the heart. To detect cardiac conditions different detection techniques are used. In this paper, a novel hybrid system combining a modified scaling technique and Wavelet technique is implemented. It is applied to enhance the accuracy of filtration, denoising and diagnosis techniques. In previous computerized diagnosis techniques, either filtration or denoising is used. However, in this system, filtration and denoising are mixed in pre-processing to give a pure signal. This research deems as the premier work to utilize, in the diagnosis phase, the time feature of each wave and its location in the ECG signal. In contrast to previous automated techniques, the proposed hybrid system is based on three factors to detect and diagnose the ECG episodes; namely amplitude, frequency and time location scaling of the ECG signal. Mixing effectively these three factors in the diagnosis phase allows the detection of more episodes, gives more accurate and faster results. As the results demonstrate, the previous computerized techniques' average detection accuracy does not exceed $80 \%$, while the proposed hybrid technique average accuracy overtakes $97 \%$ with a good average time consumption equal to 0.05 seconds. Furthermore, the proposed system overcomes some of the previous challenges and detects more new episodes that have never been diagnosed before by any automated systems. This system can help the cardiologists to take more confident decisions in their diagnoses.

Corresponding author: Mai A. Shams El-Din Teaching assistant at the Institute of Engineering and Technology, Alexandria, Egypt, mai_abdelnaby77@yahoo.com, Tel. 01114500270 


\section{INTRODUCTION}

Heart diseases are one of the most threatening diseases affecting human lives (1) . In March 2016, cardiovascular diseases caused 46\% of total deaths (2). Therefore, early diagnosis of heart diseases is very important to limit their danger.
The ECG recorded signal contains waves and intervals as shown in Figure 2. Each wave has a meaning, P-waves represent the activation (depolarization) of atria, QRS complexes show the activation behaviour of the ventricles and T-waves stand for the inactivation (repolarization) of the ventricles. PR interval means the time taken from

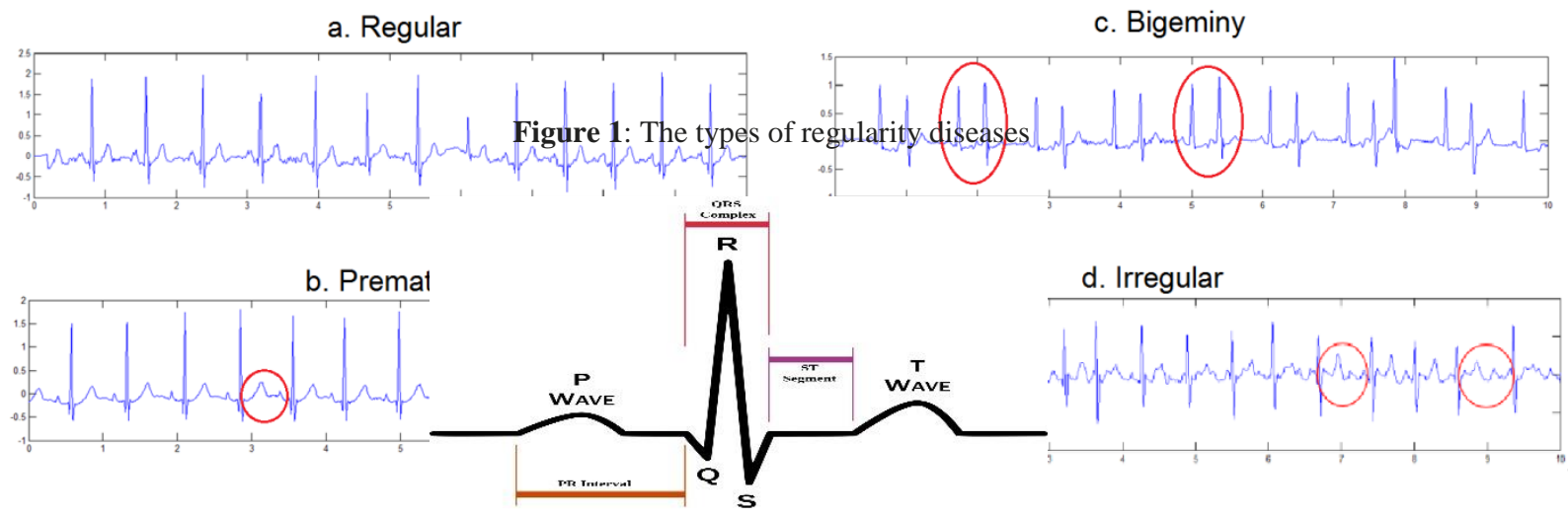

Figure 2: A sample of ECG signal with its waves and intervals

The electrical signal travels across the heart causing the heart beating rhythm (3). The normal range of heart rate is from 60 to 100 beats per minute (bpm). Less than $60 \mathrm{bpm}$ is termed bradycardia, more than $100 \mathrm{bpm}$ is termed tachycardia. Very fast, irregular, fatal, nondiscernable beats are termed ventricular fibrillation (VF) causing shock. Heartbeats have a rhythmic regular rate and any arrhythmic change is considered abnormal like premature, irregular and bigeminy (4). If less than 3 earlier beats than normal occur, it means a premature episode as shown in Figure 1 (b). If less than 3 later beats than normal occur, this means a skip episode. Irregular periodic pairs of heartbeats mean a bigeminy episode shown in Figure 1 (c). The different time between beats means irregular episodes as shown in Figure1 (d). As the figure illustrates the time between beats is an indication of many episodes. the activation of SA node till the atria are activated, QRS interval describes the time the ventricles take to be activated and ST-segment shows the time it takes from the end of the ventricular depolarization till the start of the repolarization process (3). Any change in the shape of the ECG waves is considered as an indicator for heart diseases such as large $\mathrm{T}$ waves (hyperkalemia), small $\mathbf{R}$ waves, large negative $\mathbf{S}$ waves and flat or negative $\mathrm{T}$ waves (hypokalemia). However, the present shape-based computerized technique, which is the traditional scaling technique, is based only on the amplitude of the ECG waves. The cardiologists in practical depend, in their decision, on other factors of the ECG shape alongside the peak of each wave. 
Computer-interpretation ECG (CIE) programs is considered a life-saving program. It must be smart and accurate or the physician must have enough skill and dependability to avoid using them entirely (5). Technical specifications about the various computer programs were reviewed along with numerous articles regarding accuracy data on various algorithms (6). However, the following drawbacks were pointed out. Where, there is no internationally accepted standard for computer interpretations. As a result, significant variability exists among interpretations from different manufacturers' algorithms and different variation in accuracy among the various programs (7). They have a frequent tendency to overcall atrial fibrillation, especially in elderly persons, potentially leading to inappropriate administration of harmful medications.

CIE programs tend to double-count the rate due to large $\mathrm{T}$-waves (eg, in the setting of hyperkalemia) or small R-waves. Furthermore, CIE techniques demonstrate wide variations in the false-positive $(0 \%-42 \%)$ and false-negative $(22 \%-42 \%)$ rates of diagnosis of ST-segment myocardial infarction; therefore, it is not recommended that CIEs be used as a sole means of activation of the cardiac catheterization lab. It has been roughly estimated that CIE misdiagnoses may account for up to 10,000 adverse effects or avoidable deaths worldwide annually (8).

In most previous automated ECG diagnosis researches, researchers applied either traditional scaling technique or Wavelet technique.

The works based on the traditional scaling technique $(9,10)$, used different amplitude thresholds for the detection stage to diagnose the signal. The works depended on the Wavelet transform technique $(11,12)$, applied the Wavelet decomposition to extract features of the ECG waves. However, these previous approaches suffer from the following. Firstly, the traditional scaling technique uses only the signal amplitude for interpretation of ECG electrical activity by detecting the peaks of the ECG signal. But, this technique cannot detect many abnormal episodes that depend on the time of each wave or time irregularity between beats. Secondly, applying Wavelet alone in the detection phase contributes low accuracy, as mentioned in previous researchers and assured by our results in the result section. Finally, neither Wavelet nor traditional scaling can detect cases depending on the time of the ECG signal like premature, bigeminy, Small R, Large $\mathrm{T}$ or double counting. As a result, an expert decision is the only available diagnosis for these cases.

For these reasons, this work aims to solve the problem facing cardiologists with the previous computer interpretation programs. The proposed hybrid system diagnoses a lot of new episodes that were never detected nor diagnosed by any computer program before, for example, premature and bigeminy. Furthermore, it solved the doublecounting errors because of large $T$ waves or split $R$ waves. The suggested modification overcomes the traditional scaling insufficiency by using both the time and amplitude of each wave in the diagnosis phase. This technique can calculate the rising points, the falling points and the time taken for each wave as a discriminating feature to enhance the detection accuracy. The proposed approach can offer cardiologists a highly accurate, less time consuming, inexpensive and secure system. 


\section{Materials and Methods}

In this work, a hybrid system combines the modified scaling technique and the Wavelet technique is applied. Applying the modification and combination gives better results than each one individually in denoising and diagnosing. The proposed hybrid system based on two different techniques to give a pure signal; they are the filtrations (using different types of filters) for removing any external noise and the denoising (using Wavelet transform) for deleting the body internal noise. The detection method depended on two different techniques: the modified scaling technique that depends on the amplitude and the time of ECG waves; the Wavelet transform technique that relies on the frequency analysis of the ECG signal. The modified Scaling technique not only used the amplitude of the signal but also its time, to solve the difficulties in the traditional scaling technique. The proposed modification avoids the traditional scaling errors by using the time and amplitude of each wave. The modified scaling technique can calculate the rising points, the falling points and the time taken for each wave to compare between them as a feature for waves. For example, R-waves take less time than $\mathrm{T}$ waves, applying the modified technique we can differentiate between them to cancel the doublecounting due to large $\mathrm{T}$-waves or small $\mathrm{R}$-waves as a solution to the problem mentioned before in section 1. By defining the time of each wave, the location can be defined in the whole ECG signal. If the location of a wave is so close to another, it means a split wave and it happens mostly in Rwaves. It can be cancelled by rejecting one of those R-waves.
The traditional scaling technique used only the signal amplitude for interpretation of ECG electrical activity through detecting the peaks of the ECG signal $(9,10)$, however, this technique cannot detect a lot of abnormal episodes as will be illustrated in the result section. After combining the signal time dimension to the traditional scaling, many of these abnormal episodes are detected with high accuracy and less time consumption.

Wavelet technique used for frequency analysis of the heart signal because of the confusion between noise frequencies and heartbeat frequency. It analyzes the signal frequency with different windows and different cutoff frequencies. Through the changeable window size, Wavelet can detect more features about the signal (13). The wavelet window is defined by the applied mother Wavelet, the used mother Wavelet in this work is Daubechies 4 (DB4) as it is the most suitable mother Wavelet window compatible with the ECG signal (11).

In this work, Wavelet transform technique was used in two phases, first to remove the high frequency band noises and select only the ECG signal band. Secondly, to detect the frequency band of Ventricular fibrillation because of its severity. VF cannot be detected correctly by any scaling technique as it is related to the frequency domain of the signal. It must be detected precisely to solve the alarm problem mentioned before in section 1 .

However, for an accurate detection process, it is necessary to have a neat signal to extract its features; so pre-processing is a primary step then applying the detection step on the available ECG signal as illustrated below. All 
these processes are implemented by MATLAB software code $(13,14)$.

\subsection{ECG Data}

The ECG signals data are found online by Massachusetts Institute of Technology. It is a free access source for research purposes and selected according to reference recommendations. The database consists of 180 episodes of ECG that covers all the studied episodes in this research.

\subsection{Processing for ECG signals}

ECG signals carry a lot of noise in different bands. Noise is divided into external noise like power line in the environment and internal noise like the lung sounds. Few researches focused on denoising the ECG signal from noises to get pure signals and compared between different types of filtration and denoising techniques $(11,14)$. To our knowledge, no research before tried to mix between filtration and denoising techniques in the pre-processing phase.The filtration phase has three steps: power line by using band stop filter with cutoff frequencies of $49.9 \mathrm{~Hz}$ to $50.1 \mathrm{~Hz}$; baseline wander shift through applying FIR high pass filter with cutoff frequency $1 \mathrm{~Hz}(15,16)$, and DC offset filtration to remove any surrounding noises distorting the ECG signals. The denoising phase removes any internal noises from the lung, muscles and other organs and selects only the ECG signals frequency band. The ECG frequency band exists below the $64 \mathrm{~Hz}$. In Figure 3, the detected level is used to determine the approximated signal (CA) using a Daubechies 4 (DB4) mother Wavelet transform and eliminate the detailed signals (CDs) with the high frequencies which carry almost all the noises (17). These steps give a pure signal as shown in Figure 4.

\subsection{Detection techniques for ECG signal}

A hybrid system between a modified scaling technique and Wavelet frequency transform technique is applied to detect as many accurate features as possible. Wavelet technique analyzes the frequencies carried in the ECG signal, if the signal carries a frequency above $200 \mathrm{~Hz}$, this signal is regarded as a ventricular fibrillation episode and runs a danger beep sound with red texting as an alarm. PQRST waves have frequency bands affected by the residual small irremovable noises, so their frequency analysis gives less accurate results than using the hybrid system. After the pre-processing procedure, the ECG signal passes through an adaptive gain technique to fix the threshold level, where $\mathrm{R}$ wave peaks can be detected easily. The detected peaks are filtered to remove any double-counting caused by large $\mathrm{T}$ waves or split $\mathrm{R}$ peaks using the proposed time scaling technique.

The difference between each $\mathrm{R}$ peak is calculated to get to the RR intervals, then from RR intervals the heart rate is calculated by the equation:

$$
H \cdot R=\frac{60}{\text { average of } R R \text { intervals }}
$$




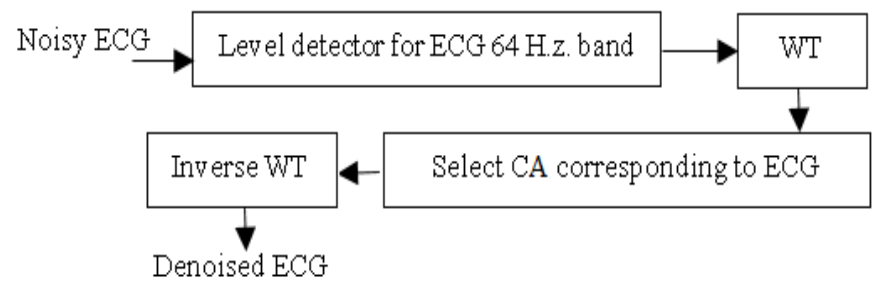

Figure 3: The wavelet (WT) denoising Technique Sequence

CA: it is a wavelet (WT) coefficient which contains much less noise than does the original signal.
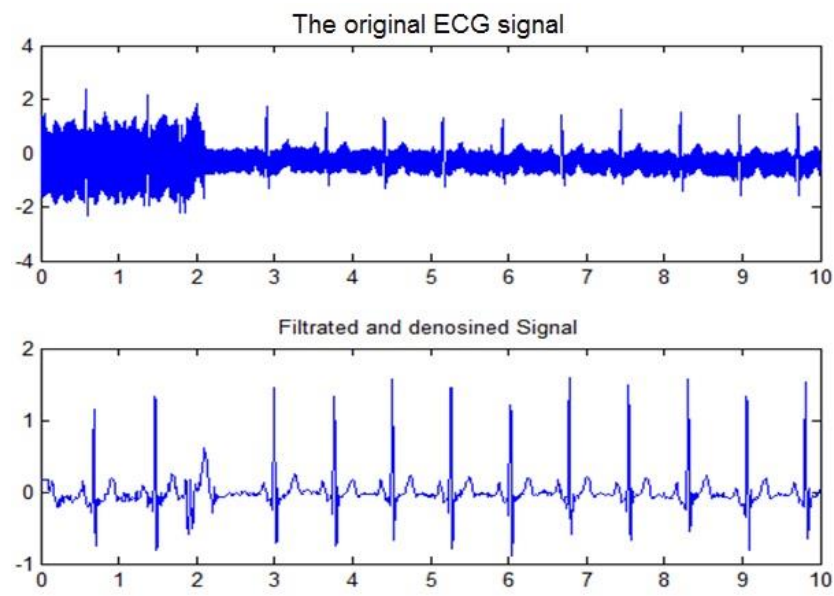

Figure 4: The ECG signal before and after filtration and denoising

The rhythmic state of the heart beating is defined by heart rate. Normal heart rate is between 60 and 100 beats per minute, bradycardia is below 60 beats per minute) and tachycardia above 100 beats per minute.

As the RR intervals are found, the regularity of the ECG signal could be determined by checking the difference between each RR intervals, if they are almost equal, it is a regular ECG signal, if not it is an irregular ECG signal with several irregularity types (shown in figure 1).

For small R-wave episodes that cannot be detected by the above threshold, the heart rate and regularity state are defined from S-waves. Large negative S-wave episodes are detected using a negative threshold after passing through the adaptive gain technique.
If $\mathrm{S}$-waves exceeds the threshold, then the ECG signal has large negative $\mathrm{S}$-waves. From those detected S-waves, heart rate and regularity state could be calculated.

The large positive $\mathrm{T}$-waves of Hyperkalemia episodes defined by finding a double-counting error detected without small Rwaves. The amplitude value of $\mathrm{T}$-waves is greater than its previous R-wave, and the timing of the Twave is located after its previous R-wave. Flat or negative $\mathrm{T}$-waves of hypokalemia episodes are identified by separating the positive values and the negative values of the ECG signal, checking the number of samples in the positive part and the negative part according to the sampling frequency. If the number of samples above the zero value is less than the samples below it, it is a flat or 
negative $\mathrm{T}$ - waves (hypokalemia) episode, if not it is a normal $\mathrm{T}$ wave.

For the last detected episode, Bigeminy is that the ECG signal is irregular but it has a periodic shape as shown in Figure 1. The first PQRS-T wave happens then an earlier P-QRS-T wave happens and they are repeated periodically. This episode is defined by checking the irregularity existence through measuring the RR interval and the interval between the next two $\mathrm{R}$ peaks. If the RR intervals are almost equal, it is a Bigeminy ECG signal.

This work has an automatic self-accuracy examination. An expert cardiologist diagnoses the episodes, and then the true diagnosis is listed in files with the same name of each episode. Comparing the true with the computerized diagnosed values, the system can calculate its accuracy.

This system measures the time consumption taken to give the diagnosis of each case. Minimizing the time consumed for diagnosis is essential in reallife cases, where the results have to show up as fast as possible on the health care monitor.

\section{Results}

The MATLAB software code runs as a batch processing for files including all episodes. Their actual diagnosis is recognized by their names as a reference for automatic accuracy determination, so each result is calculated individually. The average accuracy percentage for the proposed technique was on average $97.3 \%$ for all episodes illustrated in Table 1 . Where, Table 1 summarizes the detailed accuracy for each episode and compare the proposed approach's detection accuracy to those of the previous automated techniques. Figure 4 shows how much pure signal will be ready for the detection phase after applying both filtration and denoising technique instead of using only one technique filtration or denoising $(11,14)$.

In Table 1, the time consumption range for the novel hybrid system technique started from 0.025949 seconds to 0.199965 seconds with an average number of 0.0503004 seconds. The ventricular fibrillation episode took a long time because the alarm sound played and plotting each episode takes a few milliseconds longer. The accuracy and time consumption are measured automatically and individually.

The used episodes are categorized into three categories: normal episodes that are free of any abnormalities or errors, abnormal episodes that are with defects like arrhythmic diseases, shape or interval changes, and finally the error episodes like double counting or split R-wave.

Table 2 demonstrates the sensitivity and specificity of the proposed hybrid technique. Where, sensitivity indicates how well the proposed technique in identifying those patients with a certain disease and calculated as shown in equation 2. Specificity refers to the ability of the proposed approach to correctly identify those patients without this disease and is determined by equation 3. Where, True Positive (TP) indicates that the patient has the studied disease and the proposed technique correctly predicted. Similarly, a True Negative (TN) refers to that, the patient does not have this disease and the proposed algorithm gives negative. A False Positive (FP) represents that the patient does not have the studied disease but the proposed model indicates that it is present (predict positive). Finally, a False Negative (FN) refers 
that patient has the disease but the proposed technique results negative.

According to Table 2 results, the proposed hybrid technique has a high ability in detecting and identifying a specific disease among multiple tested ones. Furthermore, it assures that the hybrid system can precisely define both normal and abnormal episodes.

$$
\begin{aligned}
& \text { sensitivity }=\frac{T P}{T P+F N} \\
& \text { Specificity }=\frac{T N}{T N+F P}
\end{aligned}
$$

As the results illustrate, applying the modification and combination enhanced the results in denoising, diagnosing and overcomes the four shortages of previous automated methods in section 1.2. Where, not only all episodes are defined in high accuracy but also there are new episodes diagnosed and major errors are solved using the proposed hybrid automated technique. These episodes were not detected either solved before, which gives this new hybrid system the credibility in the cardiology field.

\section{Discussion}

In (18), a survey overviewed many types of ECG acquisition, compression, feature extraction, data mining algorithms, ECG analysis, and telecardiology done by different researchers until 2015. Several researchers built up their work on feature extraction and detecting the difference between waves in ECG signals. Some of them used traditional scaling techniques (9), others used a neural network to extract features $(19,20)$. While others applied the Wavelet transform technique $(11,21)$ for detecting and diagnosing ECG signals.
Wavelet technique relays only on frequency analysis. As a result, it gave less accuracy if it is implemented individually, so most of the researchers preferred to hybrid wavelet transform with another technique such as the neural network technique. It was mentioned that the hybrid system between the Wavelet technique and the neural network did not produce acceptable results and the neural network is a high time-consuming technique $(17,19)$.

Table 1 compares between the traditional scaling $(9,10)$, Wavelet technique $(11,17,21)$, and the proposed hybrid system is shown. The results proved that the novel hybrid system improved the detection accuracy and the performance where the hybrid technique produced higher accuracy in all cases. The novel hybrid system discovered the episodes that cannot be detected by the traditional scaling or the wavelet technique individually. This is because the hybrid system is based on 3 components, amplitude, frequency and time interval scaling (the newly added parameter). The amplitude factor to classify the wave by its value and if this value is normal or not. The frequency factor to recognize the presence of ECG waves and if they are detected in the normal frequency band or not. Finally, the time scaling factor to determine the time interval for each wave, not just for the wave-to-wave interval, and if it is in the normal range or not. After mixing those 3 components the whole detection picture is clearer and the diagnostic ability becomes easier and more accurate. Even though the novel hybrid technique consists of three components, this did not affect the time consumption and it has a very good processing time range as shown in Table 1. As the results shows, the total performance is calculated 
as an average only for the episodes detected by the three techniques, these episodes are bold in Table 1. The previous techniques average detection accuracy is less than $80 \%$ while the proposed technique average accuracy exceeds 97\%. The proposed hybrid system improved the detection performance, in almost all studied episodes, as shown in figure 5 .

In figure 5, the novel hybrid system provided better results compared with the traditional scaling and the Wavelet transform techniques. This results due to the mixture between the three features in the diagnosis phase. The traditional amplitude scaling technique $(9,10,15)$ had lower results due to relying only on the amplitude threshold regardless of the frequency or the time scaling factors. Besides, it applied only filtration for noise removal. The proposed technique is much better and less time consuming than the neural network that takes a lot of time in the learning stage. In the applied Wavelet technique, the detection is based only on the frequency band of the wave neglecting the shape of the wave, which makes the results less accurate in comparison with the novel hybrid system taking in care all possible factors for wave specifications.

The novel hybrid system can detect five more episodes than the previously applied techniques. These episodes were too difficult to be recognized and diagnosed by only one component. For example, Bigeminy episode could not be detected using traditional scaling technique because it is defined by its periodic irregular time interval change. It could not be detected using Wavelet transform technique because wavelet tests only frequency band for each wave in ECG. In the novel hybrid technique after using the new time scaling technique, the periodic change in time intervals is determined very well.

For the double-counting error episodes, either the large $\mathrm{T}$ or the split $\mathrm{R}$, both were considered in the traditional scaling technique as $\mathrm{R}$-wave if they were above the $\mathrm{R}$ threshold and this is a wrong diagnosis. While in the Wavelet technique, the large $\mathrm{T}$ wave or the split $\mathrm{R}$ were detected in their normal frequency band even if they were not detected at their normal ranges of amplitude_and this affects the diagnosis of the ECG signals. This error is solved easily after adding the time scaling technique as the system can differentiate between the waves by their time intervals. Where, the R-wave takes less time than the T-wave, by the different intervals the hybrid system can differentiate between the R-wave and the $\mathrm{T}$-wave. If the $\mathrm{R}$-wave is split, by location the system can cancel one of the two as a solution for the double-counting error. As for the negative or flat $\mathbf{T}$, either the $\mathrm{T}$ wave has a negative value, or it is flat with Zero value. The traditional scaling cannot distinguish that because it has a different threshold than the normal $\mathrm{T}$ or has no value to be detected by the threshold. The Wavelet technique cannot recognize the flat $\mathrm{T}$ as it has no frequency band or define the negative $\mathrm{T}$ as a normal $\mathrm{T}$ as they have the same frequency band and it can't differentiate between them. On the other hand, the novel hybrid system can solve this because of the addition of the location and time of each wave. If the $\mathrm{T}$ wave is absent in its normal location it is diagnosed as a negative or flat $\mathrm{T}$ wave episode.

Another example is the Premature, it is detected as an irregular episode in both the traditional scaling and Wavelet techniques, but in 
the novel hybrid system, after adding the location and time interval for each wave, the system can define the number of waves that were irregular not just for the whole signal. Finally, for the small Rwave, the traditional scaling cannot discover this episode as the R-wave is too small than its amplitude threshold. Wavelet technique cannot recognize this episode as it is considered as normal R-wave frequency band and these reasons incorrectly affected the diagnosis. After adding the time scaling technique the wave can be detected by its time interval and correctly detected as R-wave with small amplitude. As for Bradycardia, Tachycardia, and normal HR and the regularity episodes, they can be recognized well using traditional amplitude thresholds. However, in these episodes, the proposed hybrid approach has higher accuracy than the traditional one because of mixing filtration and denoising in the signal prepossessing, leading to clearer signal available in the detection phase.

On the other hand, these episodes impaired by using Wavelet technique individually as the R-wave presents in its normal frequency band but not with the normal heartbeats per second. These cases can be defined very well in the novel hybrid technique as the presence of the $\mathrm{R}$-wave is checked by the frequency, the number of beats by the amplitude scaling and the location of each R-wave is checked by the new time scaling parameter.

Some diseases depend on the wave shape such as the large positive $\mathrm{T}$ - waves and large negative $\mathrm{S}$-waves. In the large positive T-wave, the traditional technique detected the large $\mathrm{T}$-wave as R-waves causing double-counting errors. However, the hybrid system is much better because of its identifying for the time interval of the T-wave and its location by the addition of the time scaling parameter. Also, the hybrid system is superior to the Wavelet technique, wherein wavelet the T-wave was detected only by its normal frequency band despite its value. Finally, for the ventricular fibrillation episode, the traditional scaling technique cannot detect this episode as fibrillation relates to the frequency component of the signal not to its amplitude.

To summarize, this work is considered as premier research in mixing between different preprocessing techniques to get a better-denoised pure ECG signal as a pre-preparation step before the detection and diagnosing step. The time scaling technique in the detection and diagnosing step is presented for the first time to detect the ECG waves not only by its value or interval but also by its time and location in the whole ECG signal. Utilizing the wave time in the modified technique allows discovering of many episodes undetected by traditional scaling such as regularity (premature) and bigeminy. Furthermore, it solved the traditional errors such as double counting and split R-wave errors. For this technique, the average accuracy was $97.3 \%$ for all studied cases and the average time consumption was 0.0503004 seconds. After presenting the above results, this system should be considered a trusted technique for cardiologists upon which they can base their decisions. 


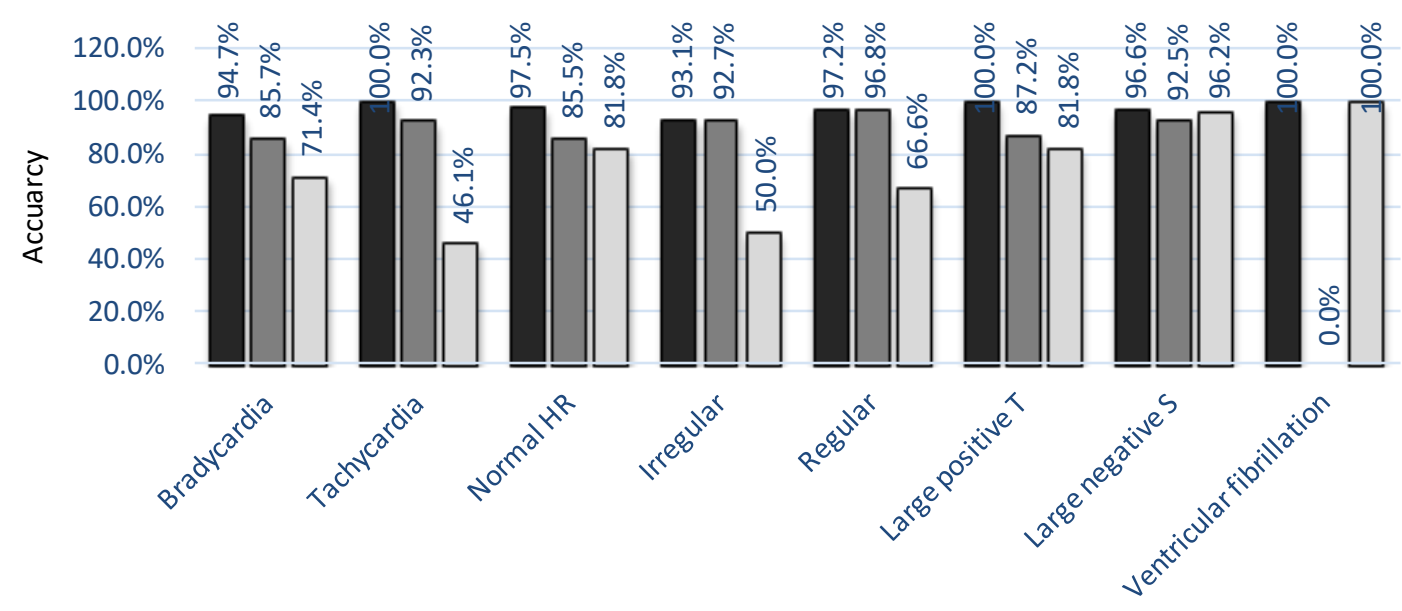

Episodes

Dercentage result of novel hybrid

$\square$ Percentage result of traditional scaling technique

$\square$ Percentage result of Wavelet technique

Figure 5: A comparison between the detection accuracy of detected episodes by the traditional scaling, Wavelet, and the proposed hybrid techniques.

Table1: Hybrid system detection accuracy for each disease and comparison between the three techniques

\begin{tabular}{|c|c|c|c|c|c|c|}
\hline Name of the episode & $\begin{array}{c}\text { Episode } \\
\text { s } \\
\text { diagnos } \\
\text { ed by } \\
\text { the } \\
\text { cardiolo } \\
\text { gist }\end{array}$ & $\begin{array}{c}\text { Episode } \\
\text { s } \\
\text { diagnos } \\
\text { ed by } \\
\text { comput } \\
\text { er }\end{array}$ & $\begin{array}{c}\text { Time } \\
\text { processing } \\
\text { (sec) }\end{array}$ & $\begin{array}{c}\text { Detection } \\
\text { accuracy } \\
\text { of novel } \\
\text { hybrid }\end{array}$ & $\begin{array}{l}\text { Detection } \\
\text { accuracy } \\
\text { of } \\
\text { traditiona } \\
\text { I scaling }\end{array}$ & $\begin{array}{c}\text { Detection } \\
\text { accuracy } \\
\text { of } \\
\text { Wavelet } \\
\text { technique }\end{array}$ \\
\hline Bigeminy & 9 & 9 & 0.033153 & $100.0 \%$ & $\begin{array}{c}\text { Not } \\
\text { detected }\end{array}$ & $\begin{array}{c}\text { Not } \\
\text { detected }\end{array}$ \\
\hline Double counting & 44 & 44 & 0.03068 & $100.0 \%$ & $\begin{array}{c}\text { Not } \\
\text { detected }\end{array}$ & $\begin{array}{c}\text { Not } \\
\text { detected }\end{array}$ \\
\hline Flat or Negative $T$ & 47 & 44 & 0.038029 & $93.6 \%$ & $\begin{array}{c}\text { Not } \\
\text { detected }\end{array}$ & $\begin{array}{c}\text { Not } \\
\text { detected }\end{array}$ \\
\hline Premature & 30 & 28 & 0.031028 & $93.3 \%$ & $\begin{array}{c}\text { Not } \\
\text { detected }\end{array}$ & $\begin{array}{c}\text { Not } \\
\text { detected }\end{array}$ \\
\hline Small R & 22 & 22 & 0.033426 & $100.0 \%$ & $\begin{array}{c}\text { Not } \\
\text { detected }\end{array}$ & $\begin{array}{c}\text { Not } \\
\text { detected }\end{array}$ \\
\hline Bradycardia & 19 & 18 & 0.0300003 & $94.7 \%$ & $85.7 \%$ & $71.4 \%$ \\
\hline Tachycardia & 35 & 35 & 0.0487501 & $100 \%$ & $92.3 \%$ & $46.1 \%$ \\
\hline Normal HR & 118 & 115 & 0.034357 & $97.5 \%$ & $85.5 \%$ & $81.8 \%$ \\
\hline Irregular & 44 & 41 & 0.048035 & $93.1 \%$ & $92.7 \%$ & $50 \%$ \\
\hline Regular & 106 & 103 & 0.043005 & $97.2 \%$ & $96.8 \%$ & $66.6 \%$ \\
\hline Large positive $T$ & 6 & 6 & 0.02715 & $100.0 \%$ & $87.2 \%$ & $81.8 \%$ \\
\hline Large negative $S$ & 58 & 56 & 0.044205 & $96.6 \%$ & $92.5 \%$ & $96.2 \%$ \\
\hline Ventricular fibrillation & 8 & 8 & 3.415536 & $100.0 \%$ & $0.0 \%$ & $100 \%$ \\
\hline Total performance & & & 0.0503004 & $97.7 \%$ & $79.1 \%$ & $74.2 \%$ \\
\hline
\end{tabular}


Table 2: The Sensitivity and Specificity of the proposed hybrid system

\begin{tabular}{|c|c|c|c|c|c|c|c|c|}
\hline \multirow{2}{*}{ Case name } & \multirow{2}{*}{$\begin{array}{c}\text { Number } \\
\text { of } \\
\text { positive } \\
\text { cases }\end{array}$} & \multicolumn{2}{|c|}{ Positive cases } & \multirow{2}{*}{$\begin{array}{c}\text { Number } \\
\text { of } \\
\text { Negative } \\
\text { cases }\end{array}$} & \multicolumn{2}{|c|}{$\begin{array}{c}\text { Negative } \\
\text { cases }\end{array}$} & \multirow{2}{*}{ Sensitivity } & \multirow{2}{*}{ specificity } \\
\hline & & $\begin{array}{l}\text { True } \\
\text { (TP) }\end{array}$ & False (FP) & & $\begin{array}{l}\text { True } \\
\text { (TN) }\end{array}$ & $\begin{array}{c}\text { False } \\
(\mathrm{FN})\end{array}$ & & \\
\hline Bradycardia & 19 & 18 & 1 & 161 & 158 & 3 & 0.86 & 0.99 \\
\hline Tachycardia & 35 & 35 & 0 & 145 & 145 & 0 & 1 & 1 \\
\hline $\begin{array}{l}\text { Ventricular } \\
\text { fibrillation }\end{array}$ & 8 & 8 & 0 & 172 & 172 & 0 & 1 & 1 \\
\hline Normal HR & 118 & 115 & 3 & 62 & 61 & 1 & 0.99 & 0.95 \\
\hline Premature & 30 & 28 & 2 & 150 & 144 & 6 & 0.82 & 0.99 \\
\hline Irregular & 44 & 41 & 3 & 136 & 134 & 2 & 0.95 & 0.98 \\
\hline Regular & 106 & 103 & 3 & 74 & 74 & 0 & 1 & 0.96 \\
\hline $\begin{array}{l}\text { Large } \\
\text { negative S }\end{array}$ & 58 & 56 & 2 & 122 & 119 & 3 & 0.95 & 0.98 \\
\hline $\begin{array}{l}\text { Large } \\
\text { positive } \mathrm{T}\end{array}$ & 6 & 6 & 0 & 174 & 174 & 0 & 1 & 1 \\
\hline $\begin{array}{l}\text { Flat or } \\
\text { Negative T }\end{array}$ & 47 & 44 & 3 & 133 & 124 & 9 & 0.83 & 0.98 \\
\hline Normal T & 127 & 118 & 9 & 53 & 50 & 3 & 0.98 & 0.85 \\
\hline Small R & 22 & 22 & 0 & 158 & 158 & 0 & 1 & 1 \\
\hline $\begin{array}{l}\text { Double } \\
\text { counting }\end{array}$ & 44 & 44 & 0 & 136 & 136 & 0 & 1 & 1 \\
\hline Bigeminy & 9 & 9 & 0 & 171 & 171 & 0 & 1 & 1 \\
\hline
\end{tabular}

\section{Acknowledgment}

Thanks to MIT-BIH database which is a free license database as a support to the new researches.

\section{References}

1. Maharani A, Sujarwoto, Praveen D, Oceandy D, Tampubolon G, Patel A. Cardiovascular disease risk factor prevalence and estimated 10-year cardiovascular risk scores in Indonesia: The SMARThealth Extend study. PLoS One. 2019;14(4):1-13.

2. Attia I. Country report Egypt - October 2014. Eur Assoc Cardiovasc Prev Rehabil. 2014;(October).

3. Enderle JD, Bronzino JD. The Biomedical Engineering Handbook. 2nd ed. 2000. $100-620$ p.
4. Malmivuo J, Plonsey R. Principles and applications of bioelectric and biomagnetic field. 1st ed. 1995. 393-401 p.

5. AlGhatrif M, Lindsay J. A brief review: history to understand fundamentals of electrocardiography. J Community Hosp Intern Med Perspect. 2012;2(1):1-5.

6. Schläpfer J, Wellens HJ. ComputerInterpreted Electrocardiograms: Benefits and Limitations. J Am Coll Cardiol. 2017;70(9):1183-92.

7. Sommer A, Tielsch JM, Katz J, Quigley HA, Gottsch JD, Javitt JC, et al. The diagnostic performance of computer programs for the interpretation of electrocardiograms. $\mathrm{N}$ Engl $\mathrm{J}$ Med. 1991;325(20):1412-7. 
8. Mele PF. The ECG dilemma: guidelines on improving interpretation. J Healthc Risk Manag. 2008;28(2):27-31.

9. Chen G, Wang X, Wan W. An ECG Rwave detection algorithm based on adaptive threshold. Int Conf Smart Sustain City Big Data. 2015;(July, Shanghai, China):145-9.

10. Priya, Singh M. MATLAB based ECG signal noise removal and its analysis. Int Conf Recent Adv Eng Comput Sci. 2015;2(December,Chandigarh, India):3-7.

11. Yadav T. Denoising and SNR Improvement of ECG Signals Using Wavelet Based Techniques. Int Conf Next Gener Comput Technol. 2016;2(October, Dehradun, India):678-82.

12. Sridhar C, Acharya UR, Fujita H, Bairy GM. Automated diagnosis of Coronary Artery Disease using nonlinear features extracted from ECG signals. IEEE Int Conf Syst Man, Cybern. 2016;(October,Budapest, Hungary):9-12.

13. Saxena S, Kumar V, Hamde S. QRS detection using new wavelets. J Med Eng Technol. 2002;26(1):7-15.

14. Hamiane M, Al-Heddi IY. Automatic Detection of Abnormalities in ECG Signals: A MATLAB Study. Int J Biol Biomed Eng. 2015;9:121-6.

15. Khan T, Sultana N, Reza RB, Mostafa R. ECG Feature Extraction in Temporal Domain and Detection of Various Heart Conditions. Int Conf Electr Eng Inf Commun Technol. 2015;2(May, Dhaka, Bangladesh):1-6.
16. Choudhry MS, Puri A, Kapoor R. Removal of baseline wander from ECG signal using cascaded Empirical Mode Decomposition and morphological functions. Int Conf Signal Process Integr Networks,. 2016;3(February,Noida, India):769-74.

17. Ranjan R, Giri VK. A Unified Approach of ECG Signal Analysis. Int J Soft Comput Eng. 2012;2(3):5-10.

18. Moses D, Deisy. A survey of data mining algorithms used in cardiovascular disease diagnosis from multi-lead ECG data. Kuwait J Sci. 2015;42(2):206-35.

19. Debnath T, Hasan MM, Biswas T. Analysis of ECG signal and classification of heart abnormalities using Artificial Neural Network. Int Conf Electr Comput Eng. 2016;9(December, Dhaka, Bangladesh):353-6.

20. Butt MM, Akram U, Khan SA. Classifying Normal Sinus Rhythm and Cardiac Arrhythmias in ECG Signals Using Statistical Features in Temporal Domain. Asia Model Symp Classifying Conf. 2015;9(september,Kuala Lumpur, Malaysia):13-6.

21. José M. Characterization of QRS Complex in ECG Signals Applying Wavelet Transform. Int Conf Mechatronics, Electron Automot Eng Charact. 2015; (November,Prague, Czech Republic):86-9. 